\title{
The role of verbal short-term memory in task selection: How articulatory suppression influences task choice in voluntary task switching
}

\author{
Christina R. B. Weywadt • Karin M. Butler
}

Published online: 22 December 2012

(C) Psychonomic Society, Inc. 2012

\begin{abstract}
The roles of verbal short-term memory (vSTM) in task selection and task performance processes were examined when individuals were asked to voluntarily choose which of two tasks to perform on each trial randomly. Consistent with previous voluntary task-switching (VTS) research, we hypothesized that VSTM would support random task selection by maintaining a sequence of previously executed tasks that would be used by a representativeness heuristic. Furthermore, because using a representativeness heuristic requires sufficient time for updating and comparison processes, we expected that vSTM would have a greater effect on task selection when more time was available. Participants completed VTS under concurrent articulatory suppression and foot tapping at short and long response-to-stimulus intervals (RSIs). Task selection in VTS was more repetitive under suppression than under foot tapping, but this effect did not vary with RSI, suggesting that vSTM does not maintain the sequence of executed tasks to guide task selection. Instead, vSTM is critical for maintaining the intended task and ensuring that it is carried out. In contrast to the finding that a working memory load impairs task performance, we found no difference in reaction times and no switch costs between suppression and foot-tapping conditions, suggesting that VSTM is not critical for task performance.
\end{abstract}

Keywords Task choice · Voluntary task switching · Articulatory suppression $\cdot$ Verbal short-term memory

C. R. B. Weywadt $(\bowtie) \cdot K$. M. Butler

Department of Psychology, University of New Mexico, MSC 03

2220, Logan Hall,

Albuquerque, NM 87131-0001, USA

e-mail: cweywadt@unm.edu

K. M. Butler

e-mail: kmbutler@unm.edu
Understanding the cognitive control mechanisms that guide task selection and task performance is important for improving individual decision making such as health behaviors. Multicomponent models of working memory suggest that the work involved in task selection and task reconfiguration can be divided between the central executive, phonological loop, and visual/spatial sketchpad (Baddeley \& Hitch, 1974). Our purpose here is to understand whether, and how, systems of the phonological loop contribute to task selection in voluntary task switching.

The phonological loop supports verbal short-term memory (vSTM) via covert articulation and has been implicated as a mediator of task performance in multitasking environments. In these studies, the task to be performed on each trial is determined by an experimenter-directed cue or instruction. To interfere with the use of covert articulation to support performance, participants are asked to repeat a task-irrelevant word aloud, in time with a metronome (for a review, see Baddeley \& Logie, 1999). Comparing task switching with and without articulatory suppression suggests that vSTM guides task switching by encoding the task cue (Baddeley, Chincotta, \& Adlam, 2001; Emerson \& Miyake, 2003; Liefooghe, Vandierendonck, Muyllaert, Verbruggen, \& Vanneste, 2005; Mayr \& Kliegl, 2000; Miyake, Emerson, Padilla, \& Ahn, 2004) and maintaining the previously executed task sequence (Bryck \& Mayr, 2005).

Cue encoding is required when the task is not directly indicated by the task cue - for example, performing a color judgment in response to a letter "C" as a task cue. Task sequence maintenance is needed when individuals must maintain previous task selections to guide current task selection. For example, Saeki and Saito (2009) used transition cues to explore the role of vSTM in task maintenance. The transition cues came in two forms: a cue to indicate that an individual should repeat the task they had performed on trial $n-1$ and a cue to indicate that individuals should switch to a new task. These researchers combined task cue with transition cues in mixed blocks and 
found that mixing costs for transition cues were significantly larger when individuals performed articulatory suppression rather than foot tapping, while switch costs were unaffected. This was interpreted as evidence that verbal resources (used up by articulatory suppression but not by foot tapping) contributed to task set maintenance.

Experimenter-instructed task switching (EITS) is valuable for understanding how individuals move between tasks and follow instructions, but EITS cannot address processes related to selection (e.g., Allport \& Wylie, 1999; Logan \& Bundesen, 2004; Mayr \& Kliegl, 2000). In a new form of task switching, voluntary task switching (VTS), the experimenter provides a performance goal for the entire session, asking participants to choose tasks randomly, trial by trial, "as though flipping a coin” (Arrington \& Logan, 2004, 2005). Switching probability $[p(\mathrm{sw})]$ across all trials is the primary measure of task choice, while the traditional switch costs are thought to reflect the demands of task switching (Arrington \& Yates, 2009; Butler, Arrington, \& Weywadt, 2011).

Current theory holds that task selection in VTS is governed by two types of processing, the representativeness and availability heuristics. The representativeness heuristic relies on expectations about the repetitions of items and frequency of transitions between different items in the "random" sequences as a guide for task selection (e.g., Arrington, 2008; Arrington \& Logan, 2004, 2005). As Rapoport and Budescu (1997) explain,

when asked to produce a binary random series whose length exceeds the capacity of STM, which we denote by $m$, the subject considers the last $m$ items already produced and then chooses the $(m+1)^{\text {th }}$ response to produce the most likely or 'locally representative' series. (p. 605)

In random generation, individuals mistakenly overrepresent how often a change should occur (e.g., Baddeley, Emslie, Kolodny, \& Duncan, 1998). In VTS, however, switch probabilities are lower than would be expected if switches were chosen randomly, suggesting that the availability of a task set also contributes to task selection. The availability heuristic bases task choices on the task that comes to mind most easily. Because the task executed on the previous trial is typically the most active in memory, reliance on the availability heuristic biases task repetitions (Arrington \& Logan, 2004, 2005).

Whether representativeness or availability is the basis for any single task selection depends on the time available for task selection and the contents of short-term memory. Task repetitions are more likely at short than at long response-to-stimulus intervals (RSIs; Arrington \& Logan, 2004). At long RSIs, more time is available for maintaining the previous sequence of executed tasks, as well as for making the comparison with a representative sequence, resulting in more frequent switching. At short RSIs, the time demands on responding prevent the coordination of maintenance, comparison, and selection processes supporting the representativeness heuristic; instead, the most available task is used as the basis for performance. Availability is determined by residual activation from previous trials and retrieval of stimulus-response (S-R) mappings based on the current stimulus. Indeed, Weaver and Arrington (2010) found that increasing the availability of S-R bindings by biasing attentional orienting influences task selection in VTS. When two univalent stimuli were presented, task choice was biased toward the stimulus that matched the identity or location maintained in working memory for a secondary task. This finding suggests that when S-R bindings are retrieved more quickly, task selection is biased toward that response (see also Arrington, 2008).

In contrast, task environments that influence memory for the previously executed sequence of task choices also influence switching. Arrington and Logan (2005, Experiment 4) found that task selection was sensitive to the number of instructed task repetitions; longer sequences of instructed task repetitions led to more switching than did shorter ones. The intervening instructed task repetitions presumably influenced the sequence of task choices maintained in working memory and, through use of the representativeness heuristic, resulted in more choices to switch to a new task.

The conceptualization of the representativeness heuristic as relying on the short-term storage of sequences of tasks, combined with the findings from the EITS literature demonstrating that remembering sequences of tasks to perform relies on vSTM, suggests that VTS will rely on vSTM to maintain the sequence of previously executed tasks. Although the availability of a task set can be influenced by information held in STM (Weaver \& Arrington, 2010), the operation of the availability heuristic is not expected to rely on vSTM. Indeed, Demanet, Verbruggen, Liefooghe, and Vandierendonck (2010) found that a concurrent working memory load that placed a large demand on articulatory processes (i.e., maintaining a sequence of words) decreased $p(\mathrm{sw})$.

\section{The present study}

Using the VTS paradigm, this experiment examines the role of the vSTM in task selection and performance. We measured $p$ (sw) and switch costs in VTS with concurrent articulatory suppression (which exhausts vSTM resources) and foot tapping (which does not require vSTM). Demanet et al. (2010, Experiment 1) similarly utilized a concurrent load, albeit with greater working memory demand, and found that the secondary task affected stimulus repetition trials more than stimulus change trials (Demanet et al., 2010). Therefore, we examined whether task selection differed on stimulus repetition and change trials.

The concurrent task was expected to reduce the reliance on the representativeness, but not the availability, heuristic 
for task selection, resulting in reduced $p(\mathrm{sw})$. Furthermore, we expected that the influence of VSTM would be greater under conditions that typically reflect a greater utilization of the representativeness heuristic. Specifically, at the long RSI, we expected the disruption of vSTM to reduce $p(\mathrm{sw})$ more than at the short RSI, reflecting the increased reliance on a representativeness heuristic.

Previous research provides no clear guidance regarding how task performance in the VTS environment will be affected by articulatory suppression. On the one hand, if task choice can be characterized as a retrieval process similar to EITS with ambiguous task cues, we might expect a similar effect on task performance; that is, articulatory suppression would increase task repetition reaction times because it interferes with the ability to maintain S-R mappings, resulting in the need for task set retrieval (Liefooghe et al., 2005). This relationship would actually predict smaller switch costs under articulatory suppression in VTS. On the other hand, when task-sequencing demands are high in EITS - for example, alternating runs - articulatory suppression does not affect switch costs (Bryck \& Mayr, 2005). To the extent that VTS has the high sequencing demands as observed in EITS, we expect that performance metrics will be unaffected by articulatory suppression.

\section{Method}

\section{Participants}

Fifty-three participants were recruited from introductory psychology courses at the University of New Mexico and were given partial course credit for their participation. Forty-eight participants were included in the final data analysis (26 females; age, $M=20$ years, $S D=2.4$; education, $M=13.1$ years, $S D=$ 1.4). Of those who were excluded from analysis, one person did not understand the directions, one person reported a diagnosis of depression, another person reported a diagnosis of a learning disability, a fourth person switched more than $95 \%$ in the noload condition, and the fifth person had fewer than four observations in one or more of the experimental cells.

Apparatus and materials

A Dell Dimensions computer running E-Prime software presented the VTS procedure (Schneider, Eschmann, \& Zuccolotto, 2002). Stimuli were the digits 1-4 and 6-9 in Courier New font. The stimuli were displayed at $7 \times 5 \mathrm{~mm}$ on a 17-in. CRT monitor. The stimuli were selected randomly on each trial and were presented just above a fixation point that was presented in the center of the screen. Responses were made on a Psychological Software Tools serial response box that has five buttons arrayed linearly.

\section{Procedure}

Participants were told that they would be performing two tasks that involved making judgments about digits, then began by practicing single-task odd/even judgments, followed by single-task high/low judgments. Responses were made with the middle and index fingers of the left and right hands. The mappings both of response hands to tasks and of keys to responses were counterbalanced across participants.

After single-task practice, participants were instructed regarding the VTS procedure. They were told to make a choice to perform one of the tasks on each trial. They were also instructed to perform the tasks equally often and in a random order, as if they were "flipping a coin" (Arrington \& Logan, 2005). Participants performed practice VTS trials while the experimenter observed and provided additional instruction, if needed.

Participants completed VTS while performing three concurrent task conditions: articulatory suppression, foot tapping, and no load. The articulatory suppression task required individuals to repeat the word "the" in time with a metronome set to $2 \mathrm{~Hz}$, while the foot-tapping condition required individuals to tap their foot in time with a metronome set to $2 \mathrm{~Hz}$. The RSI was fixed at two levels: 500 and 1,300 ms. Participants completed 2 blocks of trials (64 trials/block) in each secondary load and RSI condition, for a total of 12 blocks of trials. Each block was followed by accuracy feedback. The order of the concurrent task load conditions and the order of RSI blocks were counterbalanced across participants.

\section{Results}

Experimental trials were coded for accuracy by comparing each participant's response with the two possible correct responses. If a response matched one of the two possible responses, the trial was assigned the corresponding task code; otherwise, the trial was labeled as an error. A trial was designated as a repeat trial if the same task was executed on the previous trial and as a switch trial if the other task was executed on the previous trials.

\section{Data trimming}

Trials that began each block were excluded from the analyses. Trials with reaction times longer than $3,000 \mathrm{~ms}$ or less than $150 \mathrm{~ms}$ were excluded from the accuracy analyses. In addition to those trials, error trials and trials following errors were excluded from the $p(\mathrm{sw})$ and reaction time analyses $(11.7 \%$ of trials were excluded). Calculations of $p(\mathrm{sw})$ for stimulus repetitions were based on 13-15 trials in each load by RSI condition and were distributed similarly across conditions (of all analyzed trials, stimulus repetitions occurred on $2 \%$ of no- 
load trials under both RSIs and $1.9 \%$ of articulatory suppression trials for both RSIs, as well as $2.2 \%$ and $2 \%$ of the foottapping trials at the 500- and 1,300-ms RSIs, respectively). $P$ (sw) by load and RSI is displayed in Fig. 1. Means by condition are presented in Table 1 for $p(\mathrm{sw})$, Table 2 for reaction times, and Table 3 for accuracies.

Task choice

$P(\mathrm{sw})$ was analyzed in a 2 (RSI: 500 or $1,300 \mathrm{~ms}) \times 2$ (stimulus type: stimulus repetition or stimulus change) $\times 3$ (load: no concurrent load, concurrent articulatory suppression, or concurrent foot tapping) within-subjects ANOVA.

As was expected, there was a main effect of RSI, $F(1,47)=$ $34.0, p<.001, \eta_{\mathrm{p}}^{2}=.412$, as well as main effects of stimulus type, $F(1,47)=110.3, p<.001, \eta_{\mathrm{p}}^{2}=.701$, and load, $F(2,94)=$ $14.0, p<.001, \eta_{\mathrm{p}}^{2}=.229$. These effects were qualified by a marginally significant three-way interaction, $F(2,94)=2.82, p=$ $.06, \eta_{\mathrm{p}}=.059$.

To demonstrate the unique contribution of phonological loop resources to task choice, holding constant the need to coordinate a secondary task that also required repeated action, we compared $p(\mathrm{sw})$ for the concurrent articulatory suppression condition and the foot-tapping condition. $P(\mathrm{sw})$ was significantly less at $500 \mathrm{~ms}(.26)$ than at $1,300 \mathrm{~ms}(.32), F(1,47)=$ $31.1, p<.001, \eta_{\mathrm{p}}{ }^{2}=.398 . P(\mathrm{sw})$ was less when the stimulus repeated (.23) than when it changed (.35) from the previous trial, $F(1,47)=87.1, p<.001, \eta_{\mathrm{p}}{ }^{2}=.650 . P(\mathrm{sw})$ was significantly less with concurrent articulatory suppression (.27) than with foot tapping $(.30), F(1,47)=7.36, p=.009, \eta_{\mathrm{p}}{ }^{2}=.135$.

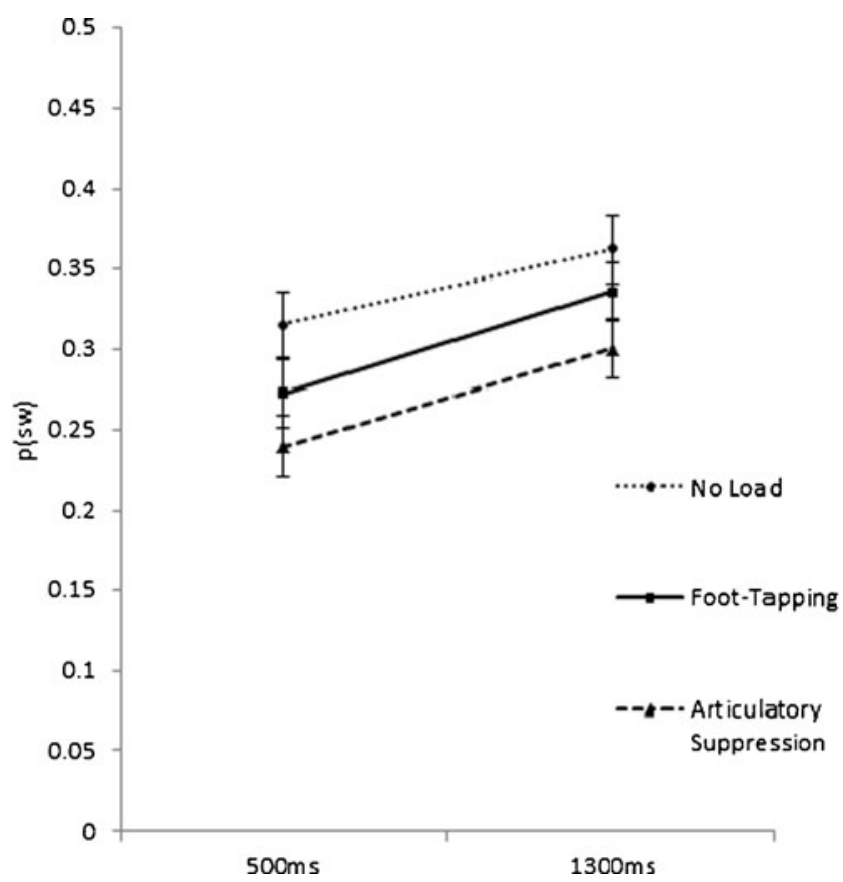

Fig. 1 Probability of switching as a function of concurrent load and response-to-stimulus interval (error bars are standard errors)
Table 1 Probability of switching by concurrent load, response-tostimulus interval (RSI), and stimulus repetition

\begin{tabular}{lll}
\hline & \multicolumn{2}{l}{ RSI } \\
\cline { 2 - 3 } & $\begin{array}{l}500 \mathrm{~ms} \\
\mathrm{M}(\mathrm{SE})\end{array}$ & $\begin{array}{l}1,300 \mathrm{~ms} \\
\mathrm{M}(\mathrm{SE})\end{array}$ \\
\hline No load & & \\
Stimulus repeated & $.268(.024)$ & $.299(.025)$ \\
Stimulus switched & $.361(.022)$ & $.426(.021)$ \\
Foot tapping & $.192(.024)$ & $.276(.023)$ \\
Stimulus repeated & $.352(.021)$ & $.395(.017)$ \\
Stimulus switched & $.183(.023)$ & $.250(.023)$ \\
Articulatory suppression & $.295(.022)$ & $.350(.020)$ \\
Stimulus repeated & & \\
Stimulus switched &
\end{tabular}

As can be seen in Table 1, $p(\mathrm{sw})$ did not differ between the articulatory suppression and foot-tapping conditions when the stimulus repeated, but when the stimulus changed, concurrent articulatory suppression led to an additional decrement in switching, $F(1,47)=4.27, p=.04, \eta_{\mathrm{p}}{ }^{2}=.083$.

Task performance

To evaluate the effects of articulatory suppression on task performance, we evaluated reaction times and accuracies in two 3 (load) $\times 2($ RSI $) \times 2$ (task transition: task repeated or task switched) ANOVAs. Analyses of reaction times and accuracies were restricted to trials with stimulus changes because there were insufficient trials for evaluating the effects on stimulus repeat trials.

\section{Reaction times}

Reaction times differed across the load conditions, $F(2,94)=$ $8.1, p=.001, \eta_{\mathrm{p}}{ }^{2}=.148$, and responding was slower for a task switch than for a task repetition, $F(1,47)=48.7, p<.001, \eta_{\mathrm{p}}{ }^{2}=$ .509 . There was no main effect of RSI $(p=.2)$, but RSI did enter into a two-way interaction with transition, $F(1,47)=$ $35.3, p<.001, \eta_{\mathrm{p}}{ }^{2}=.429$. Switch costs were smaller at the long (45 ms) than at the short (117 ms) RSI. A marginally significant three-way interaction between RSI, load, and transition was also observed, $F(2,94)=2.9, p=.06, \eta_{\mathrm{p}}{ }^{2}=.057$.

To evaluate the selective effects of phonological processes on task performance, the articulatory suppression condition was compared with the foot-tapping condition. Interestingly, reaction times were not different in the conditions, $F(1,47)=0.657$, $p=.422, \eta_{\mathrm{p}}{ }^{2}=.014$, nor did load enter into any interactions: load $\times$ RSI, $F(1,47)=2.6, p=.112, \eta_{\mathrm{p}}{ }^{2}=.053$; load $\times$ transition, $F(1,47)=2.5, p=.118, \eta_{\mathrm{p}}{ }^{2}=.051$; load $\times$ RSI $\times$ transition, $F(1,47)=2.7, p=.102, \eta_{\mathrm{p}}^{2}=.056$. Switch trials 
Table 2 Mean reaction time (in milliseconds) by concurrent load, response-to-stimulus interval (RSI), and task transition for stimulus change trials

\begin{tabular}{lll}
\hline & \multicolumn{2}{l}{ RSI } \\
\cline { 2 - 3 } & $500 \mathrm{~ms}$ & $1300 \mathrm{~ms}$ \\
& $\mathrm{M}(\mathrm{SE})$ & $\mathrm{M}(\mathrm{SE})$ \\
\hline No load & & \\
Task repeated & $726(23)$ & $750(23)$ \\
Task switched & $842(30)$ & $815(28)$ \\
Switch costs & 116 & 65 \\
Foot tapping & & $863(26)$ \\
Task repeated & $804(26)$ & $874(26)$ \\
Task switched & $920(32)$ & 11 \\
Switch costs & 116 & $839(30)$ \\
Articulatory suppression & & $898(33)$ \\
Task repeated & $768(25)$ & 59 \\
Task switched & $887(35)$ & \\
Switch costs & 119 & \\
\hline
\end{tabular}

were slower than repeat trials, $F(1,47)=31.1, p<.001, \eta_{\mathrm{p}}{ }^{2}=$ .398 , and these switch costs were significantly greater at the short RSI than at the long RSI, $F(1,47)=34.2, p<.001, \eta_{\mathrm{p}}{ }^{2}=$ 421. Reaction times did not differ significantly across RSIs, $F$ $(1,47)=2.4, p=.121, \eta_{\mathrm{p}}^{2}=.05$.

\section{Accuracy}

Accuracy varied across the three load conditions, $F(2,94)=$ $15.8, p<.001, \eta_{\mathrm{p}}^{2}=.252$, and load interacted with transition, $F(2,94)=13.5, p<.001, \eta_{\mathrm{p}}{ }^{2}=.224$. Comparing the

Table 3 Accuracy by concurrent load, response-to-stimulus (RSI), and task transition for stimulus change trials

\begin{tabular}{lll}
\hline & \multicolumn{2}{l}{ RSI } \\
\cline { 2 - 3 } & $500 \mathrm{~ms}$ & $1300 \mathrm{~ms}$ \\
& $\mathrm{M}(\mathrm{SE})$ & $\mathrm{M}(\mathrm{SE})$ \\
\hline No load & & \\
Task repeated & $99.5 \%(0.2)$ & $99.8 \%(0.1)$ \\
Task switched & $89.3 \%(1.4)$ & $92.5 \%(0.9)$ \\
Switch costs & $10.2 \%$ & $7.3 \%$ \\
Foot tapping & & \\
Task repeated & $98.5 \%(0.4)$ & $99.7 \%(0.1)$ \\
Task switched & $86.5 \%(1.4)$ & $90.3 \%(1.0)$ \\
Switch costs & $12.0 \%$ & $9.4 \%$ \\
Articulatory suppression & & $99.1 \%(0.3)$ \\
Task repeated & $99.0 \%(0.3)$ & $87.1 \%(1.4)$ \\
Task switched & $83.2 \%(1.8)$ & $12.0 \%$ \\
Switch costs & $15.8 \%$ & \\
\hline
\end{tabular}

articulatory suppression condition with the foot-tapping condition, accuracy was lower in the articulatory suppression condition than in the foot-tapping condition, $F(1,47)=11.7$, $p=.001, \eta_{\mathrm{p}}{ }^{2}=.199$, and this effect was greater for task switch than for task repeat trials, $F(1,47)=12.4, p=.001, \eta_{\mathrm{p}}{ }^{2}=.209$. Task repeat trial accuracy was similar regardless of the secondary load condition, but task switch trials were significantly less accurate under articulatory suppression, $t(47)=3.5, p=$ .001 . The effect of secondary load was not influenced by RSI, $F(2,94)=0.556, p=.576, \eta_{\mathrm{p}}{ }^{2}=.012$, and did not interact with RSI and transition, $F(2,94)=0.534, p=.588, \eta_{\mathrm{p}}{ }^{2}=.011$. Replicating previous work, with more time to prepare, task switch trials became more accurate, $F(1,47)=19.8, p<.001$, $\eta_{\mathrm{p}}{ }^{2}=.296$.

\section{Discussion}

Disrupting vSTM decreased switching, but this occurred independently of the amount of time available for selection. This supports a postselection account of the effect and converges with the view that VSTM maintains task representations prior to stimulus presentation (Saeki \& Saito, 2004). In EITS, this effect is reflected in decreased switch costs when phonological resources interfere with the retrieval of a new S-R mapping, a process that is unnecessary for task repetitions. In VTS, the disruption of phonological resources interferes with the maintenance of the selected task, causing the most available task to be more likely to be performed, resulting in less switching.

We found no evidence that phonological resources were being used to support the representativeness heuristic through the maintenance of a sequence of previous task choices. The increase in switching at longer preparation intervals, an effect thought to be mediated, in part, by greater reliance on the representativeness heuristic (Arrington \& Logan, 2005), was not influenced by articulatory suppression. Drawing strong conclusions from the absence of an interaction in a single experiment is always tenuous; however, this same pattern was observed in three additional experiments (Weywadt, 2012). If the representativeness heuristic is used to guide task selection in VTS, it is not verbally mediated. Similarly, Baddeley et al. (1998) found that vSTM is not necessary to support random behavior. This finding is also in line with the recently proposed chain-retrieval model of VTS, which suggests that task selection is based, in part, on a prospective plan (Vandierendonck Demanet, Liefooghe, \& Verbruggen, 2012). In this view, the decrease in switching under articulatory suppression simply reflects interference in the retrieval of the prospective plan for the task to be performed.

When the stimulus repeated, switching was equally likely with foot tapping (.23) and concurrent articulation (.22). This suggests that phonological loop resources do not influence 
switching when the environment biases a task repetition. Demanet et al. (2010) found that a secondary working memory load caused a greater decrement in switching when the stimulus repeated, as compared with when the stimulus changed, and argued that the requirement to inhibit the task repetition responses when the stimulus repeated put greater demands on central executive resources. The availability of more general resources determines whether switching will occur when stimuli repeat, perhaps through suppression of a correct, although repetitive, response that was easily retrieved.

In VTS, task set reconfiguration and/or task execution processes are better captured by the performance metrics of reaction times and accuracies than by switch probabilities (Arrington, 2008; Arrington \& Yates, 2009). Articulatory suppression caused a small (3\%) decline in the accuracy of task switch trials, as compared with foot tapping, but did not influence reaction times on either task switch or task repeat trials. Unlike Liefooghe et al. (2005), we did not find that articulatory suppression reduced switch costs. Therefore, we did not find evidence that, in VTS, S-R mappings are maintained in VSTM and, thus, speed task repetitions. Our findings also suggest that the substantial increase in reaction time switch costs in a VTS task under a concurrent working memory load that was observed by Demanet and colleagues (2010) is likely to be the results of demands on working memory, rather than vSTM. Miyake et al. (2004) found that in EITS, switch costs were affected by articulatory suppression only when performance required first retrieving the meaning of the task cue; when the task cue explicitly stated the task to be performed, switch costs were not affected by articulatory suppression. Perhaps unsurprisingly, then, when the task cue is internally generated, the interpretation of that task cue does not require VSTM, and reaction time switch costs are not affected.

Finally, when we consider the role of preparation interval, the RT and accuracy results converge with those of Bryck and Mayr (2005). These researchers used RSIs of 50 and $250 \mathrm{~ms}$. If we consider just our shortest RSI condition, $500 \mathrm{~ms}$, we see the same general pattern that they observed: faster but more error-prone responding under articulatory suppression. Interpreting this pattern as reflecting a speed-accuracy trade-off rather than a processing difference, those researchers concluded that phonological processes were not necessary to support task switching, but only task sequencing. Our findings qualify this conclusion. At the longer RSI of $1,300 \mathrm{~ms}$, reaction times remain the same under concurrent articulation, as compared with foot tapping, while accuracy still declines. It may be that phonological processes only come into play for task set reconfiguration at longer preparation intervals - in part, because of the time limitation itself. Articulation takes time to store information, and at short RSIs, the process may be unable to support retrieval or activation of S-R mappings.
Furthermore, the verbal mediation of retrieval and activation of S-R mappings at longer RSIs appears to influence the accuracy of those mappings.

Author Note The authors thank Kate Arrington and Eric Ruthruff for insightful discussions of this work. We would also like to thank Erik Altmann and anonymous reviewers for their feedback. Data collection was supported by Bryan Franks and Celatha Jane Grove, whom we thank.

This work was completed in partial fulfillment of the dissertation requirements for Christina R. B. Weywadt. Portions of this work were presented at the 2011 Annual Meeting of the Psychonomic Society in Seattle, WA.

\section{References}

Allport, A., \& Wylie, G. (1999). Task-switching: Positive and negative priming of task-set. In G. W. Humphreys, J. Duncan, \& A. Treisman (Eds.), Attention, space, and action: Studies in cognitive neuroscience (pp. 273-296). New York, NY US: Oxford University Press.

Arrington, C. M. (2008). The effect of stimulus availability on task choice in voluntary task switching. Memory \& Cognition, 36(5), 991-997. doi:10.3758/MC.36.5.991

Arrington, C. M., \& Logan, G. D. (2004). The cost a voluntary task switch. Psychological Science, 15, 610-615. doi:10.1111/j.09567976.2004.00728.x

Arrington, C. M., \& Logan, G. D. (2005). Voluntary task switching: Chasing the elusive homunculus. Journal of Experimental Psychology: Learning, Memory, and Cognition, 31, 683-702. doi: 10.1037/0278-7393.31.4.68

Arrington, C. M., \& Yates, M. M. (2009). The role of attentional networks in voluntary task switching. Psychonomic Bulletin \& Review, 16(4), 660-665. doi:10.3758/PBR.16.4.660

Baddeley, A., Chincotta, D. M., \& Adlam, A. (2001). Working memory and the control of action: Evidence from task switching. Journal of Experimental Psychology: General, 130, 641-657. doi:10.1037/0096-3445.130.4.641

Baddeley, A., Emslie, H., Kolodny, J., \& Duncan, J. (1998). Random generation and the executive control of working memory. The Quarterly Journal Of Experimental Psychology A: Human Experimental Psychology, 51A(4), 819-852. doi:10.1080/ 027249898391413

Baddeley, A. D., \& Hitch, G. J. (1974). Working memory. In G. Bower (Ed.), The psychology of learning and motivation vol. 8 (pp. 4790). San Diego, CA: Academic Press.

Baddeley, A. D., \& Logie, R. H. (1999). Working memory: The multiple-component model. In A. Miyake, P. Shah, A. Miyake, \& P. Shah (Eds.), Models of working memory: Mechanisms of active maintenance and executive control (pp. 28-61). New York, NY US: Cambridge University Press.

Bryck, R. L., \& Mayr, U. (2005). On the role of verbalization during task set selection: Switching or serial order control? Memory \& Cognition, 33(4), 611-623.

Butler, K. M., Arrington, C. M., \& Weywadt, C. (2011). Working memory capacity modulates task performance but has little influence on task choice. Memory \& Cognition, 39(4), 708-724. doi:10.3758/s13421-010-0055-y

Demanet, J., Verbruggen, F., Liefooghe, B., \& Vandierendonck, A. (2010). Voluntary task switching under load: Contribution of topdown and bottom-up factors in goal-directed behavior. Psychonomic Bulletin \& Review, 17(3), 387-393. doi:10.3758/PBR.17.3.387 
Emerson, M. J., \& Miyake, A. (2003). The role of inner speech in task switching: A dual- task investigation. Journal of Memory and Language, 48(1), 148-168. doi:10.1016/S0749-596X(02)00511-9

Liefooghe, B., Vandierendonck, A., Muyllaert, I., Verbruggen, F., \& Vanneste, W. (2005). The phonological loop in task alternation and task repetition. Memory, 13(5), 550-560. doi:10.1080/ 09658210444000250

Logan, G. D., \& Bundesen, C. (2004). Very clever homunculus: Compound stimulus strategies for the explicit task-cuing procedure. Psychonomic Bulletin \& Review, 11(5), 832-840. doi:10.3758/BF03196709

Mayr, U., \& Kliegl, R. (2000). Task-set switching and long-term memory retrieval. Journal of Experimental Psychology: Learning, Memory, and Cognition, 26, 1124-1140.

Miyake, A., Emerson, M. J., Padilla, F., \& Ahn, J. (2004). Inner speech as a retrieval aid for task goals: The effects of cue type and articulatory suppression in the random task cuing paradigm. Acta Psychologica, 115(2-3), 123-142. doi:10.1016/j.actpsy.2003.12.004

Rapoport, A., \& Budescu, D. V. (1997). Randomization in individual choice behavior. Psychological Review, 104(3), 603-617. doi:10.1037/0033-295X.104.3.603
Saeki, E., \& Saito, S. (2004). Effect of articulatory suppression on taskswitching performance: Implications for models of working memory. Memory, 12, 257-271. doi:10.1080/09658210244000649

Saeki, E., \& Saito, S. (2009). Verbal representation in task order control: An examination with transition and task cues in random task switching. Memory \& Cognition, 37, 1040-1050. doi:10.3758/MC.37.7.1040

Schneider, W., Eschmann, A., \& Zuccolotto, A. (2002). EPrime reference guide. Pittsburgh, PA: Psychology Software Tools.

Vandierendonck, A., Demanet, J., Liefooghe, B., \& Verbruggen, F. (2012). A chain-retrieval model for voluntary task switching. Cognitive Psychology, 65, 241-283. doi:10.1016/ j.cogpsych.2012.04.003

Weaver, S. M., \& Arrington, C. M. (2010). What's on your mind: The influence of the contents of working memory on choice. The Quarterly Journal Of Experimental Psychology, 63, 726-737. doi:10.1080/17470210903137180

Weywadt, C. R. B. (2012). The role of Phonological Loop Resources in Task Choice and Task Performance (Unpublished doctoral dissertation). Albuquerque: University of New Mexico. 